\section{Environmental effects of urbanization - the role of urban designers}

There is increasing epidemiological evidence to the effects of urbanization on human well-being. Studies have shown that urbanization has a direct bearing on health as it creates crowding, air pollution, traffic fatalities, emotional stress and coronary heart diseases. Medium-size cities (in the $100,000-500,00$ range) and not the mega-cities seem more afflicted with diseases associated with crowding (tuberculosis, respiratory illnesses, measles, common cold, etc.). Urban dwellers experience increased morbidity to air pollutionrelated illnesses. However, when groups were controlled for income levels, rich/upper class urban and rural dwellers showed no differences in morbidity levels due to air pollution (primarily, Bronchitis). Rural males of $15-44$ years of age show the highest risk of being involved in traffic related fatalities.

Urban dwellers appear more prone to emotional disorders, but it is hard to say whether cultural differences between urban and rural dwellers had anything to do with it (for example, urban dwellers are more likely to seek medical help for emotional stresses than rural residents). Urban dwellers are also more susceptible to coronary heart diseases than rural ones, yet lifestyles that lead to such differences (sedentary occupation, fatty/highsodium content food, stress) are catching up in the rural areas as well.

Urban environments do appear to induce biochemical responses in humans. Increased serum cholestrol levels in blood, increased adrenalin secretion, leading to short term coronary damage etc. These are biochemical responses to anxiety, stress and competitiveness which are features of urban environments.

Urban environments are full of unhealthy stimuli, high exposure to which make it harder for people to carry out normal tasks. The decline in efficiency is, at least in part, not due to the specific effects of each stress, but to changes in the general state of alertness of people. "If alertness produce high arousal in persons, it can persist even long after they leave the environment which caused it." (Broadbent, 1976: 283-84)
At the same time, it must be kept in mind that human sensory mechanism is capable of wide range of adaptations which occur either consciously or unconsciously, if the environmental conditions are not optimum. This adaptation presents special problems for setting environmental standards. Often environmental standards are set not in relation to human biological capacities, but with regard to economy and technological possibilities. Thus, for example, one foot-candle (10 lux) of light was considered sufficient for most work situations at the turn of the twentieth century while $500-1000$ lux (50-100 foot-candles) is required by today's lighting codes. A room temperature of $15^{\circ} \mathrm{C}$ was considered ideal for buildings in the 1900s (Hopkinson, 1976: 75) but today's ASHRAE Standards (ASHRAE, 1992) stipulates $18-25^{\circ} \mathrm{C}$, depending on the seasons. These changes in environmental standards came about not because human beings have progressively weaker vision or difficulty in coping with colder environments, but because the economic conditions of society demand such high standards and modern technologies enable their achievement.

It is in this context that we should approach environmental changes brought about by urbanization. To complicate matters, the effects of environmental change are not immediately felt by humans. There is a lag-time involved between cause and effect in the human-environment interaction. Even when environmental changes exceed the "threshold of complaint", it takes a while before human beings exposed to the situation start complaining. The challenge in environmental design is to detect 'thresholds of complaint' long before they actually occur (cf. Hopkinson, 1976: 76).

The progress of environmental effects upon human beings is something like the following:

"Threshold of Complaint" $\rightarrow$ loss of efficiency $\rightarrow$ short-term biological/ psychological damage ' $\rightarrow$ long-term (permanent) damage.

"The main gap in our knowledge is that we do not understand sufficiently what happens when the human being is subjected to stresses well below the breakdown point and maybe below the threshold of complaint" (Hopkinson, 1976: 80). 
Since there is a time lag between environmental cause and human health effect, and the "thresholds of complaint" vary by individuals and societies, it is proposed that environment-sensitive design ought to focus on Quality-of-Life (QoL) enhancement as a holistic proposition. Liu (1975) identifies 5 components of QoL:

$$
\begin{aligned}
& \text { * Economic Component } \\
& \text { * Political Component } \\
& \text { * Environmental Component } \\
& \text { * Health and Education } \\
& \text { * Social Component }
\end{aligned}
$$

The environmental component is the primary action arena of urban designers and planners. On the physical side of this component, the following sub categories can be considered essential for urban QoL: Air Pollution, Visual Pollution, Noise, Waste Disposal, Water Pollution, Climate and Comfort and receational facilities.

\section{References}

ASHRAE, (1992) ANSI/ASHRAE 55-1992, Standard 55: Thermal Environmental Conditions for Human Occupancy, Atlanta, GA.: ASHRAE

Broadbent, DE, (1976) "Environment and performance," in, Harrison, G.A. \& J. B. Gibson, (eds.), Man in Urban Environments, Oxford: Oxford University Press pp. 247-273

Hopkinson, RG, (1976) "Urban man in buildings," in, Harrison, GA and Gibson, JB (eds.) Man in Urban Environments, Oxford: Oxford University Press, pp. 74-107

Liu, Ben-Chieh, (1975) Quality of Life Indicators in U.S. Metropolitan Areas, 1970: A Comprehensive Assessment. Report Prepared by the Midwest Research Institute for the U.S. Environmental Protection Agency, Washington, DC

R. Emmanuel, Editor 